\title{
Variational states for the spin-Peierls system
}

\author{
Holger Frahm* and John Schliemann ${ }^{\dagger}$ \\ Institut für Theoretische Physik, Universität Hannover D-30167 Hannover, Germany
}

(Received 6 January 1997; revised manuscript received 3 March 1997)

\begin{abstract}
We introduce a family of Jastrow pair product states for quasi-one-dimensional spin systems. Depending on a parameter they interpolate between the resonating valence-bond ground state of the Haldane-Shastry model describing a spin liquid and the (dimerized) valence-bond solid ground states of the Majumdar-Ghosh spin chain. These states are found to form an excellent basis for variational studies of Heisenberg chains with next-nearest-neighbor interactions and bond alternation as realized in the spin-Peierls system $\mathrm{CuGeO}_{3}$. [S0163-1829(97)04930-8]
\end{abstract}

\section{INTRODUCTION}

Following the recent discovery of a spin-Peierls transition in the inorganic compound $\mathrm{CuGeO}_{3},{ }^{1}$ there has been growing theoretical interest in this instability of one-dimensional spin chains. It has been proposed in Refs. 2 and 3 that frustrating next-nearest-neighbor (NNN) interactions in addition to an explicitly broken translational invariance due to lattice dimerization is necessary to obtain a consistent description of the experimental data. This leads to the following spin- $\frac{1}{2}$ Hamiltonian:

$$
\mathcal{H}=\sum_{j=0}^{N-1}\left\{\left[1+(-1)^{j} \delta\right] \mathbf{S}_{j} \cdot \mathbf{S}_{j+1}+\alpha \mathbf{S}_{j} \cdot \mathbf{S}_{j+2}\right\} .
$$

For $\delta=0$, the model is invariant under translations by one lattice site. This case has been investigated in detail and includes the nearest-neighbor Heisenberg chain, $\alpha=0$, where the complete spectrum can be obtained by means of the Bethe ansatz: ${ }^{4}$ the ground state is that of a spin liquid, has a vanishing spin gap, and algebraically decaying correlations at $T=0$. For $\delta=0, \alpha=\frac{1}{2}$, the Hamiltonian (1.1) becomes that of the Majumdar-Ghosh (MG) model: $:^{5}$ here the system has a gap $^{6}$ and the exact ground state is known to be a product of nearest-neighbor singlet pairs showing a twofold degeneracy:

$$
\begin{gathered}
\left|\psi_{\mathrm{VB}}^{(1)}\right\rangle \propto[0 ; 1] \cdots[(N-2) ;(N-1)], \\
\left|\psi_{\mathrm{VB}}^{(2)}\right\rangle \propto[1 ; 2] \cdots[(N-1) ; 0]
\end{gathered}
$$

$\left([a ; b]=(1 / \sqrt{2})\left(|\uparrow\rangle_{a}|\downarrow\rangle_{b}-|\downarrow\rangle_{a}|\uparrow\rangle_{b}\right)\right.$ denotes the singlet state formed by the spins on sites $a$ and $b$ ). For intermediate values of the NNN interaction the model (1.1) remains gapless for $\alpha<\alpha_{c} \approx 0.2411$ (Refs. 7, 8, and 3) with its low energy sector described by an effective level $k=1 \mathrm{SU}(2)$ WessZumino-Witten (WZW) conformal field theory. Increasing $\alpha$ beyond the "conformal point" $\alpha_{c}$ the NNN coupling becomes marginally relevant, producing an exponentially small gap $\Delta \propto \exp \left[\operatorname{const} /\left(\alpha-\alpha_{c}\right)\right]$. In this phase the system is spontaneously dimerized. The properties of the system for $\alpha>1$ have recently been discussed in Ref. 9. Extending the discussion to general couplings in the $\alpha-\delta$ plane, the system has gap above a dimerized ground state for any nonzero $\delta,^{10}$ with the valence-bond ground states (1.2) on the line $2 \alpha+\delta=1 .^{11}$ Going to larger $\delta$ the Hamiltonian (1.1) corresponds to a ladder system of two coupled Heisenberg chains. ${ }^{12}$ In addition to numerical studies, several mean-field theories have been proposed for the Hamiltonian (1.1). ${ }^{13}$ In this framework physical properties have been calculated giving reasonable agreement with the experimental data available for energy gaps, Raman spectra, and the susceptibility of $\mathrm{CuGeO}_{3}$ and hence further support the Hamiltonian (1.1) as a model for this substance.

In this paper we propose a family of variational states for the ground state and low lying triplet of the Hamiltonian (1.1). While it will not be possible within this variational approach to compute the thermodynamical quantities mentioned above, it turns out that the states proposed are excellent approximations to the true ground state of the system throughout the parameter region of interest here, namely $2 \alpha+\delta \leqslant 1$. Hence they can be used to obtain very good variational bounds on energies and may provide a better understanding of the role of quantum fluctuations in these systems.

In fact, variational states have already been applied successfully to some of the systems mentioned above: an easy way to incorporate two-particle correlations in a variational state is to consider a Jastrow pair product wave function. Specifically, the state

$$
\begin{gathered}
\left|\psi_{0}^{N, M, J}\right\rangle=\sum_{n_{1}, \ldots, n_{M}} \psi\left(\left\{n_{i}\right\}\right) \prod_{i=1}^{M} S_{n_{i}}^{-}|\uparrow \cdots \uparrow\rangle, \\
\psi\left(\left\{n_{i}\right\}\right)=\prod_{i=1}^{M} g\left(n_{i}\right) \prod_{i<j} d\left(n_{i}-n_{j}\right)^{2},
\end{gathered}
$$

with $d(n)=\sin (\pi n / N), \quad g(n) \propto \exp [2 \pi i(J / N) n]$, and $M=J$ $=N / 2$ has been found to reproduce the ground state energy of the nearest-neighbor Heisenberg chain of $N$ sites with remarkable accuracy. ${ }^{14}$ Furthermore, Eq. (1.3) captures the essence of the spin-spin correlations in this system. States of the form (1.3) span a large part of the Hilbert space including the ground state of the Haldane-Shastry (HS) spin chain with long range exchange interactions $J_{k l} \propto 1 / \sin ^{2}[\pi(k-l) / N]{ }^{15}$ The spectrum of this model gives a representation of the 
$k=1 \mathrm{SU}(2)$ WZW conformal field theory ${ }^{16}$ (note that the next-nearest-neighbor interaction in the HS model is very close to $\alpha_{c}$ ).

The spin-Peierls system and ladderlike models on the other hand have been studied using short range resonatingvalence-bond (RVB) states as ground states and soliton states interpolating between the two singlet bond configurations (1.2) for the excitations. ${ }^{17}$

In the following section we introduce variational states depending two free parameters and prove that they are eigenstates of the total spin of the system. In Sec. II B we show that these states contain the valence-bond states (1.2) and the Jastrow state (1.3) as certain limits. Their relation to Gutzwiller projected states of spin- $\frac{1}{2}$ fermions is discussed in Sec. II C. Finally, we apply the variational states to the spinPeierls system (1.1) in Sec. III.

\section{THE VARIATIONAL STATES}

\section{A. Construction}

Let $C_{1}$ and $C_{2}$ be two parallels with polar angles $\theta$ and $\pi-\theta$, respectively, on the two-dimensional sphere $S^{2}$. For even $N$ we choose $N / 2$ equidistant points labeled by even numbers $0,2,4, \ldots,(N-2)$ on $C_{1}$ and similarly $N / 2$ points labeled by odd numbers $1,3,5, \ldots,(N-1)$ on $C_{2}$ such that their azimuthal angles satisfy

$$
\frac{2 \pi}{N}(1-2 \rho)=\varphi_{2 i+1}-\varphi_{2 i}
$$

for $i \in\{0, \ldots,(N-2) / 2\}$. This defines a lattice on $S^{2}$ characterized by the parameters $\theta$ and $\rho$ consisting of two identical linear sublattices and obeying periodic boundary conditions in the azimuthal direction. The lattice points $\vec{a}$ can be described in terms of polar coordinates on the sphere, i.e., $\vec{a}=\left(\cos \varphi_{a} \sin \theta_{a}, \sin \varphi_{a} \sin \theta_{a}, \cos \theta_{a}\right)$. Alternatively, they may be parametrized by spinor components $u_{a}$ $=\cos \left(\theta_{a} / 2\right) \exp \left(l \varphi_{a} / 2\right), v_{a}=\sin \left(\theta_{a} / 2\right) \exp \left(-\imath \varphi_{a} / 2\right)$. Given two points $a, b \in S^{2}$ we construct the spinor product

$$
d(a, b)=\left(u_{a} v_{b}-u_{b} v_{a}\right)
$$

that has the following properties:

$$
\begin{gathered}
|d(a, b)|^{2}=\frac{1}{4}\|(a-b)\|^{2}, \\
\arg [d(a, b)]=\tan ^{-1}\left(\frac{\sin \left[\left(\theta_{a}+\theta_{b}\right) / 2\right] \sin \left[\left(\varphi_{a}-\varphi_{b}\right) / 2\right]}{\sin \left[\left(\theta_{a}-\theta_{b}\right) / 2\right] \cos \left[\left(\varphi_{a}-\varphi_{b}\right) / 2\right]}\right),
\end{gathered}
$$

where $\|\cdot\|$ stands for the Euclidean norm in $R^{3}$.

Now let each lattice point carry a spin $\mathbf{S}_{n}$ with $S_{n}=\frac{1}{2}$. In the Hilbert space of these $N$ spins we consider the following states [ $d$ given by Eq. (2.2)]:

$$
\begin{aligned}
\left|\psi^{N, M, J}(\theta, \rho)\right\rangle \propto & \sum_{\left|\left\{n_{i}\right\}\right|=M} \prod_{i} e^{l(2 \pi / N) J n_{i}}\left(\prod_{j} d^{2}\left(n_{i}, n_{j}\right)\right) \\
& \times S_{n_{i}}^{-}|\uparrow \cdots \uparrow\rangle .
\end{aligned}
$$

Here $|\uparrow \cdots \uparrow\rangle$ is the ferromagnetically ordered state with all spins parallel. The sum extends over all possibilities to select $M$ out of $N$ lattice sites and invert their spins. The sets of the selected sites are denoted by $\left\{n_{i}\right\}$. Each of those spin-product states contributes with an amplitude that consists of a product of single-site phase factors and a product of Jastrow-like two-site factors, depending on the underlying lattice, namely the parameters $\theta$ and $\rho$. The spinor components on the $n$th lattice site may be rewritten up to a common factor as $u_{n} \propto \exp \left\{+l(\pi / N)\left[n+(-1)^{n} w\right]\right\}, \quad v_{n} \propto \exp \{-l(\pi / N)[n$ $\left.\left.+(-1)^{n} w\right]\right\}$ with $w=\rho+1 \kappa$ and $\kappa=-(N / 2 \pi) \ln [\cot (\theta / 2)]$. Hence, the states defined in Eq. (2.4) depend analytically on a complex parameter expressing a dimerized structure of the lattice and will be referred to as dimerized Jastrow states (DJS's). Periodic boundary conditions require the parameter $J$ to be taken integer. Requiring the DJS's to have a definite total spin restricts $J$ even further: Clearly, Eq. (2.4) are eigenstates of the $z$ component of the total spin with eigenvalue $N / 2-M$. To show that they are eigenstates of the total spin we rewrite $\mathbf{S}^{2}$ as $\Sigma \mathbf{S}_{n}^{2}+\Sigma_{n<m}\left(S_{n}^{+} S_{m}^{-}+S_{n}^{-} S_{m}^{+}+2 S_{n}^{z} S_{m}^{z}\right)$. Obviously $\Sigma_{n} \mathbf{S}_{n}^{2}\left|\psi^{N, M, J}(\theta, \rho)\right\rangle=\frac{3}{4} N,\left|\psi^{N, M, J}(\theta, \rho)\right\rangle$ and

$$
\begin{aligned}
\sum_{n<m} 2 S_{n}^{z} S_{m}^{z}\left|\psi^{N, M, J}(\theta, \rho)\right\rangle= & \frac{1}{4}(M(M-1)+(N-M) \\
& \times(N-M-1)-2(N-M) M) \\
& \times\left|\psi^{N, M, J}(\theta, \rho)\right\rangle
\end{aligned}
$$

For integer $J$ with $M \leqslant J \leqslant N-M$ this gives, together with the results of the Appendix,

$$
\begin{aligned}
\mathbf{S}^{2}\left|\psi^{N, M, J}(\theta, \rho)\right\rangle= & \left(\frac{N}{2}-M\right)\left(\frac{N}{2}-M+1\right) \\
& \times\left|\psi^{N, M, J}(\theta, \rho)\right\rangle .
\end{aligned}
$$

Hence, under the above condition the state $\left|\psi^{N, M, J}(\theta, \rho)\right\rangle$ is an SU(2) highest weight state with total spin $S=N / 2-M$ for arbitrary values of the parameters $\theta, \rho$. In particular, choosing $M=J=N / 2$ one obtains a singlet, $M=N / 2-1$, $J \in\{N / 2-1, N / 2, N / 2+1\}$ gives three triplet states with $S^{z}=1$, etc.

\section{B. Limiting cases}

From Eq. (2.2) one finds that the amplitudes of the wave functions (2.4) will be complex in general. These "chiral", spin states are believed to arise in two-dimensional spin systems due to frustrating interactions ${ }^{18}$ (see also Sec. III A below). Choosing $w \in R$, i.e., $\theta=\pi / 2$, all lattice sites lie on the equator of the sphere and the Jastrow factors in Eq. (2.4) become all real: now the relative phases of the amplitudes are determined by the one-site factors alone. In this case, some of the states discussed in the Introduction can be obtained by properly choosing $\rho$ :

(i) $w=0$ : Provided that $M-1 \leqslant J \leqslant N-M+1$, the DJS

$$
\left|\psi_{\mathrm{HS}}^{N, M, J}\right\rangle:=\left|\psi^{N, M, J}\left(\frac{\pi}{2}, 0\right)\right\rangle
$$

are eigenstates of the Haldane-Shastry model ${ }^{15}$ 


$$
H_{\mathrm{HS}}=\sum_{\substack{n, m=0 \\ n<m}}^{N-1} \frac{1}{\{(N / \pi) \sin [(\pi / N)(n-m)]\}^{2}} \mathbf{S}_{n} \mathbf{S}_{m}
$$

with the eigenvalues

$$
E_{N, M, J}=\frac{1}{3}\left(\frac{\pi}{N}\right)^{2}\left(\frac{N}{8}\left(N^{2}-1\right)+M\left(M^{2}-1\right)-3 M J(N-J)\right) .
$$

The ground state is given by the singlet, the first excitations by the triplet with $M=N / 2-1, J=N / 2$.

In the thermodynamic limit the two spin correlation functions have been evaluated exactly by Gebhard and Vollhardt. ${ }^{19}$ For the singlet state of Eq. (2.7) they have obtained

$$
\lim _{N \rightarrow \infty}\left\langle\psi_{\mathrm{HS}}^{N, N / 2, N / 2}\left|\mathbf{S}_{k} \mathbf{S}_{k+n}\right| \psi_{\mathrm{HS}}^{N, N / 2, N / 2}\right\rangle=(-1)^{n} \frac{3}{4} \frac{\operatorname{Si}(\pi n)}{\pi n} .
$$

This result is based on an alternative formulation of the DJS that is discussed below.

(ii) $w=\frac{1}{2}$. Here an even-numbered lattice site coincides with the next higher site. It is

$$
\left|\psi^{N, N / 2, N / 2}\left(\frac{\pi}{2}, \frac{1}{2}\right)\right\rangle \propto\left|\psi_{\mathrm{VB}}^{(1)}\right\rangle .
$$

The singlet of the DJS at $w=1 / 2$ is a valence-bond state: next neighbors are coupled to singlets. Equation (2.11) is easily proved by calculating the scalar product of both sides. Similarly, we have $\left|\psi^{N, N / 2, N / 2}(\pi / 2,-1 / 2)\right\rangle \propto\left|\psi_{\mathrm{VB}}^{(2)}\right\rangle$ and

$$
\begin{aligned}
& \left|\psi^{N, N / 2-1, N / 2}\left(\frac{\pi}{2}, \frac{1}{2}\right)\right\rangle \propto \sqrt{\frac{2}{N}} \sum_{k=0}^{N / 2-1}[0 ; 1] \cdots|\uparrow\rangle_{2 k} \\
& \times|\uparrow\rangle_{2 k+1} \cdots[(N-2) ;(N-1)] \text {. }
\end{aligned}
$$

DJS with higher total spin cannot be cast in a comparably simple form at $w=1 / 2$ due to the more complicated structure of the remaining amplitudes.

The two valence-bond singlets span the ground state space of the Majumdar-Ghosh model ${ }^{5}$

$$
H_{\mathrm{MG}}=\sum_{n=0}^{N-1}\left(\mathbf{S}_{n} \mathbf{S}_{n+1}+\frac{1}{2} \mathbf{S}_{n} \mathbf{S}_{n+2}\right) .
$$

Recently, Nakano and Takahashi have generalized this Hamiltonian to a variety of models with interactions of arbitrary range that have the same property ${ }^{20}$ In the thermodynamic limit these models have a finite gap for excitations over the twofold degenerate ground state leading to spin-spin correlations that decay exponentially at large distances. This is in contrast to the Haldane-Shastry model which has no gap and according to Eq. (2.10) correlations decaying algebraically.

Hence the DJS's with $\theta=\pi / 2$ and $M=J=N / 2$ interpolate between a "resonating-valence-bond" singlet for $\rho=0$ and the nearest-neighbor "valence bond solid" described by Eq. (1.2) for $\rho=\frac{1}{2}$. Despite their essentially distinct properties these states have a remarkably large overlap. In Fig. 1 the

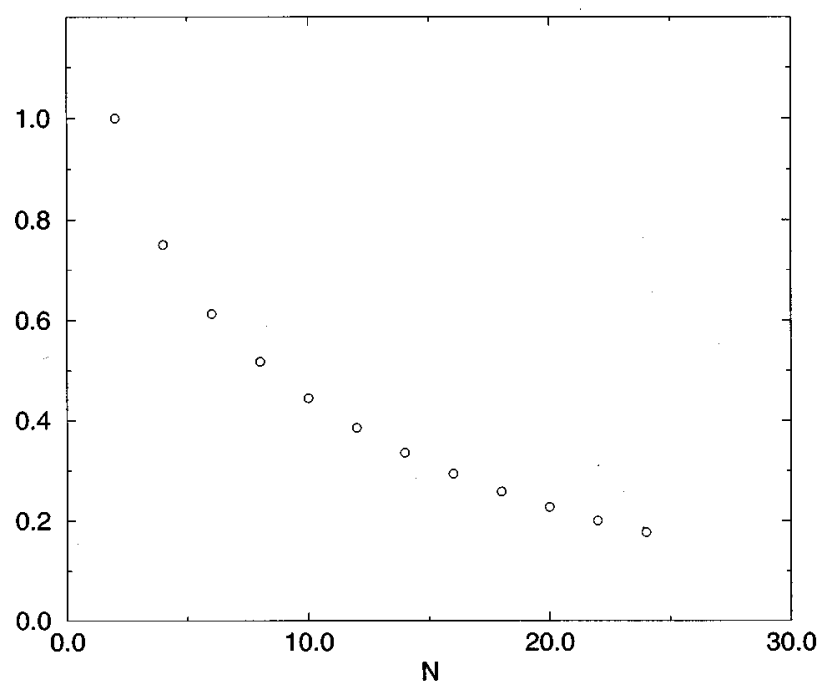

FIG. 1. Overlap $\left|\left\langle\psi^{N, N / 2, N / 2}(\pi / 2,0) \mid \psi^{N, N / 2, N / 2}(\pi / 2,1 / 2)\right\rangle\right|^{2}$ between the ground state of the Haldane-Shastry model and the valence-bond state.

squares of the overlaps as computed numerically are plotted for systems up to $N=24$. At $N=24$ the singlet subspace has the dimension 208012 , so that a square overlap of 0.17 should be regarded as quite large.

It would be interesting if one could find a Hamiltonian interpolating between one of the models given in Ref. 20 and the Haldane-Shastry model, so that the singlet DJS is always the exact ground state.

(iii) $\theta \rightarrow 0$. For $\theta \rightarrow 0$ and finite $\rho$ the chains $C_{1}, C_{2}$ are drawn to the poles of the sphere. In Eq. (2.4) all amplitudes and consequently the normalization sum become zero. In this limit tensor products of Haldane-Shastry type states arise. In the simplest case, the singlet DJS, one obtains for even N/2

$$
\lim _{\theta \rightarrow 0}\left|\psi^{N, N / 2, N / 2}(\theta, \rho)\right\rangle \propto\left|\psi_{\mathrm{HS}}^{N / 2, N / 4, N / 4}\right\rangle \otimes\left|\psi_{\mathrm{HS}}^{N / 2, N / 4, N / 4}\right\rangle,
$$

The first factor in the tensor product refers to $C_{1}$, the second to $C_{2}$. Other DJS's can also be examined by expliciting their dependence on the parameters $\theta$ and $\rho$.

\section{Relation to the Gutzwiller wave function}

It is worthwhile noticing that the DJS's can also be formulated analogous to the Gutzwiller wave function. ${ }^{21}$

Let $a_{n \sigma}^{+}, a_{n \sigma}$ be canonical creation and annihilation operators on the $n$th site for spin- $\frac{1}{2}$ particles with $S_{n}^{z}=\sigma$ and $|0\rangle$ the vacuum of the system. For $M=J=N / 2$ one can use the following construction: With the definition

$$
b_{k \sigma}^{+}(w)=\frac{1}{\sqrt{N}} \sum_{n=0}^{N-1} e^{\imath k\left(n+(-1)^{n} w\right)} a_{n \sigma}^{+},
$$

we have

$$
\begin{aligned}
\left|\psi^{N, N / 2, N / 2}(\theta, \rho)\right\rangle \propto & \mathcal{P} b_{k_{0} \uparrow}^{+}(w) \cdots b_{k_{N / 2-1} \uparrow}^{+}(w) \\
& \times b_{k_{0} \downarrow}^{+}(w) \cdots b_{k_{N / 2-1} \downarrow}^{+}(w)|0\rangle
\end{aligned}
$$


with $k_{n}=-\pi / 2+\pi / N+(2 \pi / N) n$ and $\mathcal{P}$ being the Gutzwiller projector excluding double occupancies, i.e., $\mathcal{P}=\prod_{n=0}^{N-1}\left(1-n_{n \uparrow} n_{n \downarrow}\right), n_{n \sigma}=a_{n \sigma}^{+} a_{n \sigma}$. Equation (2.16) can be proved using similar arguments as in Ref. 15. So the DJS's are deformed Gutzwiller wave functions characterized by a complex parameter $w$. Analytical results concerning the two-site correlations have been found for $w=0 .{ }^{19,22}$ For nonzero $w$ the dimerization leads to complications that cannot be resolved following these methods.

An obvious generalization is to construct wave functions of the above type with arbitrary filling. Starting from states without double occupancies on the equidistant lattice as considered in Ref. 23, one can introduce double occupancies and dimerization while keeping the Jastrow form of the states. ${ }^{24}$ Again they can be constructed to be highest weight states of the total spin. Since empty and doubly occupied lattice sites do not contribute to the spin, there are large additional possibilities of varying the wave functions in the case of general filling and strength of the Gutzwiller projection.

\section{APPLICATION TO THE SPIN-PEIERLS SYSTEM}

We now use the DJS as variational ansatz for the low lying states of the model (1.1). As mentioned above, the analytical methods of Refs. 19 and 22 cannot be applied to the dimerized system, hence the results presented below were obtained by numerical evaluation of the relevant matrix elements for system sizes up to $N=26$ lattice sites.

\section{A. Ground state properties}

For the ground state of the model we have used the singlet DJS $(M=J=N / 2)$ as a variational ansatz. The numerical results can be summarized as follows.

For $2 \alpha+\delta \leqslant 1$ the expectation value of the Hamiltonian is minimized by real $w$, i.e., $\theta=\pi / 2$ with $\rho$ varying from 0 to $1 / 2,2 \alpha+\delta=1$ corresponds to a valence-bond state $(w=1 / 2) .^{11}$ On the other hand, for $2 \alpha+\delta \geqslant 1$ we find $\rho=1 / 2$ and varying $\theta$. For $\alpha=\delta=0$ the minimum is given by the Haldane-Shastry ground state $(w=0)$. The exact ground state energy per spin is known to be $-\ln 2+1 / 4 \simeq-0.443147$ for an infinite system, the variational value is $-(3 / 4) \operatorname{Si}(\pi) /(\pi) \simeq-0.442177$ from (2.10). In Table I we present the extrapolation to an infinite system of the variational ground state energies for various values of $\alpha$ and $\delta$. Comparison with data from numerical diagonalization obtained by Chitra et al. ${ }^{25}$ shows excellent agreement: the variational energies per spin differ by only about $10^{-3}$ from diagonalization values except for the last row of Table I. In general, the quality of the singlet DJS as a variational ansatz is found to decrease for values of $\alpha$ exceeding 0.5. Hence, we have found an effective one-parametric variational wave function that gives an excellent approximation of the ground state energy of the model considered within a large area of its parameters, in particular for any antiferromagnetic NNN couplings $\alpha$ with $2 \alpha+\delta \leqslant 1$. Note that in this case the optimum singlet DJS (2.4) has real amplitudes. For $2 \alpha+\delta>1$ they become properly complex: this case therefore is called a chiral phase. For $\delta \neq 0$ the translational invariance of the system is explicitly broken leading to a dimerized variational ground state with finite $\rho$. For $\delta=0$ and small values of the NNN coupling $0 \leqslant \alpha \leqslant \alpha^{*}$, the variational energy is minimized for $w=0$. A transition to a dimerized ground state is observed at the "critical" value of $\alpha^{*} \simeq 0.2716 \pm 0.0002$ for $N \rightarrow \infty$. For $\alpha>\alpha^{*} \rho$ becomes finite. In the absence of the alternating term, the Hamiltonian $(\delta=0)$ of the system is invariant under translation by a single lattice site, while the states (2.4) do not have such a symmetry for $w \neq 0$. This leads one to consider the ansatz

$$
\left|\psi^{N}(\rho)\right\rangle \propto\left|\psi^{N, N / 2, N / 2}\left(\frac{\pi}{2}, \rho\right)\right\rangle+\left|\psi^{N, N / 2, N / 2}\left(\frac{\pi}{2},-\rho\right)\right\rangle
$$

with $N$ chosen even. This construction corresponds to the lattice momentum of the ground state of finite systems obtained in Ref. 26. With the states (3.1) one observes slight, but numerically significant, improvements of the ground state energy as shown in Table II. Furthermore, the parameter $\rho$ remains zero for $0 \leqslant \alpha \leqslant \alpha^{* *}$ with $\alpha^{* *} \simeq 0.1737$ \pm 0.0002 , which is much smaller than the $\alpha^{*}$ mentioned above. In Fig. 2 we present the ground state energy per spin as a function of $\alpha$, in Figs. 3 and $4 \mathrm{NN}$ and NNN correlations calculated within the states (3.1) are plotted. These diagrams agree very well with the corresponding figures given in Ref. 26.

Spin-spin correlations beyond those entering the expression for the ground state energy show the correct long distance asymptotics $\left\langle\mathbf{S}_{0} \mathbf{S}_{n}\right\rangle \propto(-1)^{n} / n$ as long as $\rho=0$ (2.10), which corresponds to the massless regime $\alpha \lesssim \frac{1}{4}, \delta=0$ described by the WZW conformal field theory (see, e.g., Ref. 7). Nonzero $\rho$ leads to a suppression of long range correlations. The system sizes that we have analyzed numerically do not allow one, however, to study the dependence of the correlation length on $\rho$. Only on the line $2 \alpha+\delta=1$, where the states show perfect dimerization, are the correlations read off trivially.

\section{B. Excitations}

The results of the previous section suggest using DJS with higher spin as variational ansatz for excitations of our model. Unfortunately the situation is not as clear as before. Here we

TABLE I. Variational ground state energies per spin for $N \rightarrow \infty$. The last column shows numerical data from Ref. 25 .

\begin{tabular}{lccc}
\hline \hline$\alpha$ & $\delta$ & $E_{S}^{\mathrm{var}}(\alpha, \delta, \infty)$ & $E_{0}^{\mathrm{diag}} / N$ \\
\hline 0.2411 & 0.00 & -0.40160 & -0.401866 \\
0.2411 & 0.04 & -0.40793 & -0.409051 \\
0.2411 & 0.16 & -0.44237 & -0.442862 \\
0.2411 & 0.32 & -0.49673 & -0.496844 \\
0.2500 & 0.00 & -0.40008 & -0.40045 \\
0.2500 & 0.35 & -0.50721 & -0.50727 \\
0.2500 & 0.20 & -0.46242 & -0.46329 \\
0.4000 & 0.50 & -0.56550 & -0.56611 \\
0.4800 & 0.10 & -0.41272 & -0.41281 \\
0.5500 & 0.10 & -0.41519 & -0.41610 \\
0.2000 & 0.80 & -0.67601 & -0.67613 \\
0.3000 & 0.80 & -0.67896 & -0.67966 \\
0.4800 & 0.80 & -0.68897 & -0.69256 \\
\hline \hline
\end{tabular}


TABLE II. Ground state energies per spin at $\delta=0$ and $N \rightarrow \infty$. The second column shows variational results obtained from Eq. (2.4), the third from Eq. (3.1). The data in the last column are taken again from Ref. 25.

\begin{tabular}{lccc}
\hline \hline$\alpha$ & $E_{S}^{\mathrm{var}}(\alpha, 0, \infty)$ & $E_{S, k=0}^{\mathrm{var}}(\alpha, 0, \infty)$ & $E_{0}^{\mathrm{diag}} / N$ \\
\hline 0.20 & -0.40852 & -0.40880 & -0.40885 \\
0.25 & -0.40008 & -0.40045 & -0.40045 \\
0.30 & -0.39158 & -0.39240 & -0.39284 \\
0.40 & -0.37919 & -0.37924 & -0.38028 \\
\hline \hline
\end{tabular}

concentrate on the case $\delta=0$ and mention that the eigenstates of the Haldane-Shastry model provide a good description of low lying states for $0 \leqslant \alpha \leqslant 0.3$.

From analytical and numerical studies this model is known to be gapless for $\alpha \leqslant \alpha_{c}$. As predicted by conformal field theory, at the "conformal point" $\alpha_{c}$ is defined by the occurrence of many degeneracies in addition to the usual SU(2) symmetry. Okamoto and Nomura examined the spectrum of Eq. (1.1) for $\delta=0$ in finite systems by numerical diagonalization. ${ }^{8}$ They found a linear dependence of the energy of the ground state and the two first excitations (triplet, singlet) on $\alpha$ in the above intervall. From the condition, that the two excitations should degenerate, they obtained a precise value for the conformal point as $\alpha_{c}=0.2411 \pm 0.0001$. Within the concept of DJS, there is only one singlet corresponding to the ground state, but in the HS case $(w=0)$ an additional singlet can be derived from the triplet excitation by using the Yangian symmetry of this model. ${ }^{27}$

The Yangian of the Haldane-Shastry model is generated by the total SU(2) spin or "level-0 operators" $Q_{0}^{\alpha}=\Sigma S_{n}^{\alpha}$ and the "level-1-operators" $Q_{1}^{\alpha}=\sum_{m \neq n} \cot [(\pi / N)(m$ $-n)] \varepsilon^{\alpha \beta \gamma} S_{m}^{\beta} S_{n}^{\gamma}$. We do not repeat any details of this symmetry algebra and its representations here. In the HS model the lowest excitation for an even number of spins is given by the triplet DJS $\left|\psi_{\mathrm{HS}}^{N, N / 2-1, N / 2}\right\rangle$. This state degenerates with a singlet that can be obtained by applying $Q_{1}^{-}=Q_{1}^{x}-{ }_{l} Q_{1}^{y}$ on $\left|\psi_{\mathrm{HS}}^{N, N / 2-1, N / 2}\right\rangle$ and projecting onto the singlet space, i.e.,

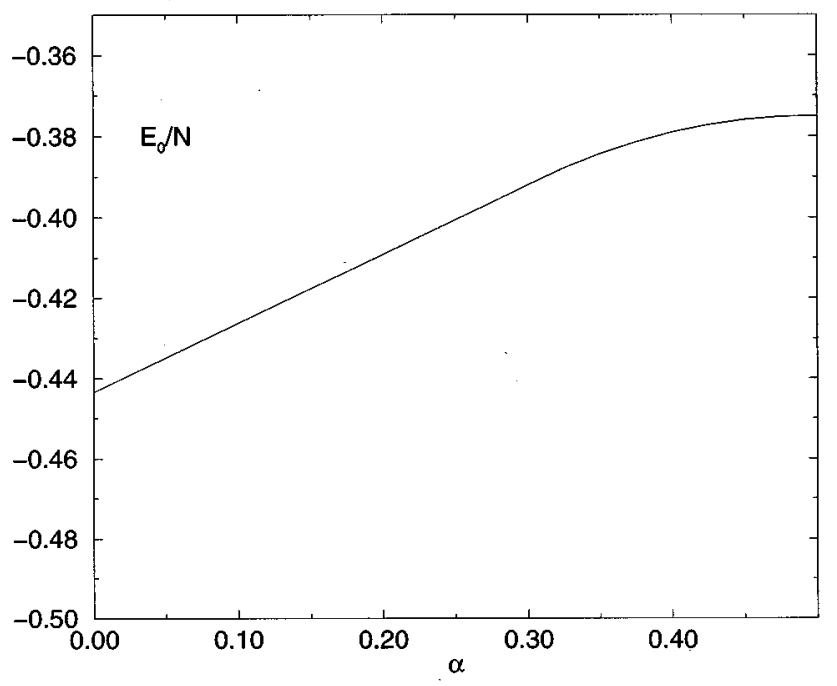

FIG. 2. Variational ground state energy per spin at $\delta=0$ as a function of $\alpha$.

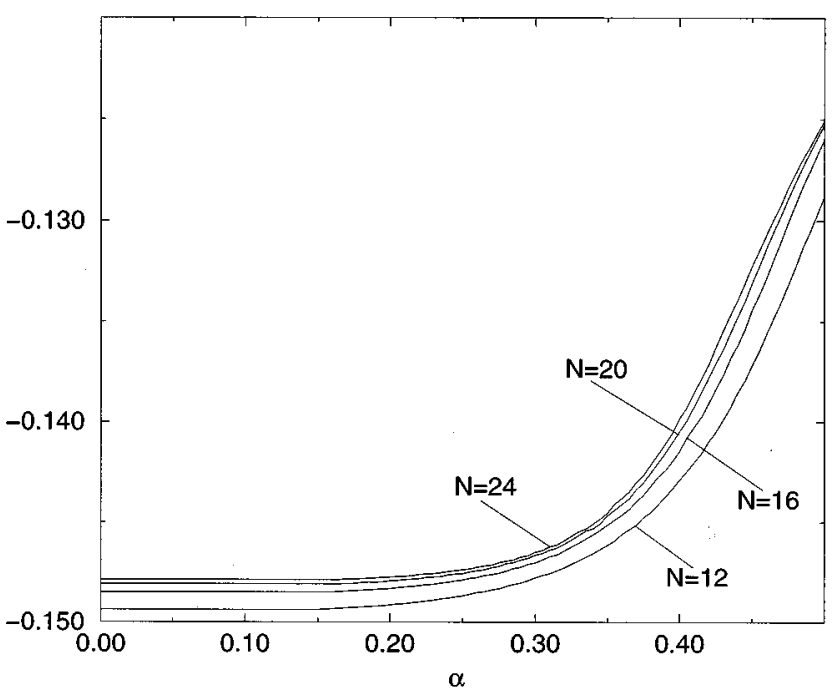

FIG. 3. Nearest-neighbor spin correlations $\left\langle S_{n}^{z} S_{n+1}^{z}\right\rangle$ $=1 / 3\left\langle\mathbf{S}_{n} \mathbf{S}_{n+1}\right\rangle$ in the variational ground state of Eq. (1.1) for $\delta=0$ as functions of $\alpha$ calculated from Eq. (3.1) for different system sizes $N$.

$$
\left|\psi_{\mathrm{HS}}^{S^{*}}\right\rangle \propto\left(2-\mathbf{Q}_{0}^{2}\right) Q_{1}^{-}\left|\psi_{\mathrm{HS}}^{N, N / 2-1, N / 2}\right\rangle
$$

As mentioned before, the minimum expectation value of the Hamiltonian on the line $\delta=0$ is given by the HS ground state for $\alpha \leqslant \alpha^{*}$. So we evaluated the Hamiltonian also for the two excited HS states above. Note that in this ansatz there is no variational parameter included, because Eq. (3.2) is not defined for $w \neq 0$. In Fig. 5 the difference of these energies with the ground state value given in Fig. 2 is plotted. For $\alpha \leqslant 0.3$ there is good agreement with the corresponding data of Ref. 8. At some $\alpha=\widetilde{\alpha}$ the singlet and triplet energies coincide. Table III shows values for $\tilde{\alpha}$ in finite systems, which can be extrapolated to $N \rightarrow \infty$ smoothly giving

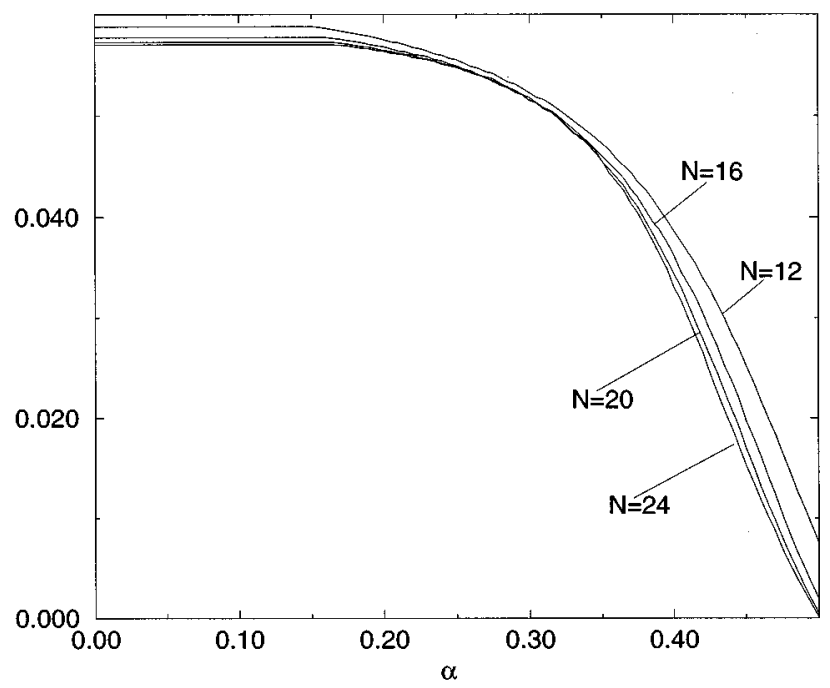

FIG. 4. Next-nearest-neighbor spin correlations $\left\langle S_{n}^{z} S_{n+2}^{z}\right\rangle$ $=1 / 3\left\langle\mathbf{S}_{n} \mathbf{S}_{n+2}\right\rangle$ of the variational ground state of Eq. (1.1) for $\delta=0$ as functions of $\alpha$ calculated from Eq. (3.1) for different system sizes $N$. 


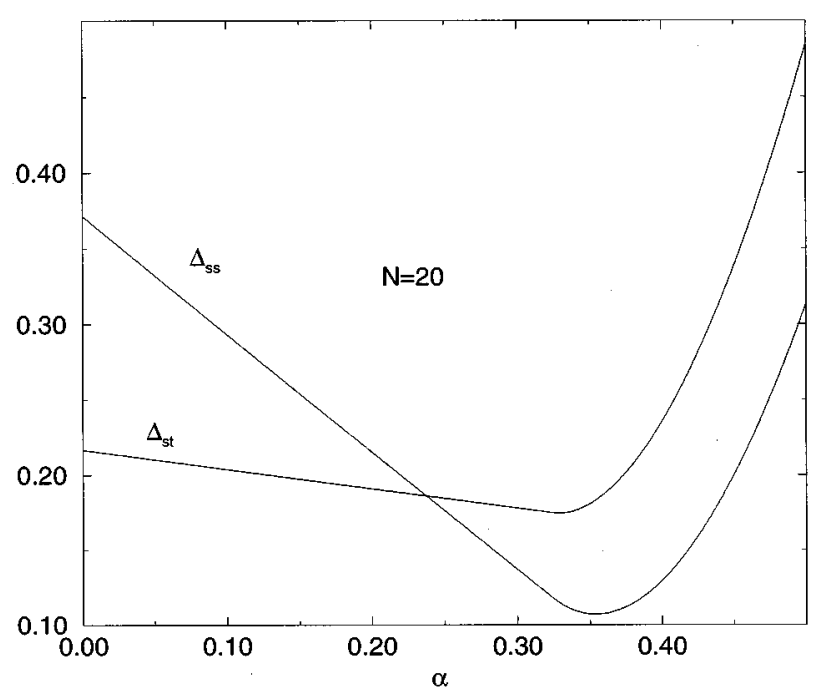

FIG. 5. Energy gap between the variational ground state and the lowest triplet $\Delta_{s t}$ and the singlet (3.2) $\Delta_{s s^{*}}$ for $\delta=0$ as a function of $\alpha$ for $N=20$. The degeneracy determines our estimate of the conformal point $\alpha_{c}$.

$\tilde{\alpha}=0.2368 \pm 0.0002$. This is remarkably close to the value $\alpha_{c}=0.2411 \pm 0.0001$ obtained from numerical diagonalization.

\section{CONCLUSIONS}

We have constructed a family of variational states which contain excellent approximations to the ground state of the model (1.1) for a large range of its parameters. The states are strictly spin singlets and can be characterized by a single complex parameter. Moreover, in Sec. III we have demonstrated that for $\delta=0$ and $0 \leqslant \alpha \leqslant 0.3$ the ground state and lowest excitations of our model can be described amazingly well by eigenstates of the HS model. For $\delta \neq 0$ the variational ansatz reproduces the explicit dimerization of the model. ${ }^{10}$ For vanishing bond alternation $\delta=0$ the ground state of the finite system has a well-defined lattice momentum $k_{s} \in\{0, \pi\}$ (Ref. 26) while the lowest excitations (triplet, singlet) have $k_{t}, k_{s} * \pi-k_{s}(\bmod 2 \pi)$. In the thermodynamic limit the excitation gap of the triplet vanishes for $\alpha \leqslant \alpha_{c}$, while for larger $\alpha$ the singlet degenerates with the ground state of the finite system. The momenta of these two states differ by $\pi$. Consequently, in this two-dimensional
TABLE III. Location of the degeneracy $\tilde{\alpha}$ between singlet and triplet excitation for different $N$.

\begin{tabular}{cc}
\hline \hline$N$ & $\tilde{\alpha}$ \\
\hline 10 & 0.23967 \\
12 & 0.23876 \\
14 & 0.23820 \\
16 & 0.23785 \\
18 & 0.23757 \\
20 & 0.23739 \\
22 & 0.23722 \\
$\infty$ & \\
& 0.2368 \\
\hline \hline
\end{tabular}

ground state space linear combinations exist that represent dimerized states. This is expressed within our variational approach by the fact that even for finite systems dimerized states (with $\rho \neq 0$ ) are good approximations of the ground state. The corresponding translational invariant states (3.1) lead to small improvements to the ground state energy, but reproduce the spin correlations between nearest and nextnearest-neighbors very well.

As mentioned above a generalization of this variational state to similar systems away from half filling is straightforward. This may allow for similar studies of the $\mathrm{Zn}$-doped compound $\mathrm{Cu}_{1-x} \mathrm{Zn}_{x} \mathrm{GeO}_{3}$ (Ref. 28) when the $\mathrm{Zn}$-sites are treated as static spin-0 sites in the spin chain.

\section{ACKNOWLEDGMENTS}

The authors thank F. H. L. Eßler and M. Takahashi for useful discussions on this topic. This work was supported by the Deutsche Forschungsgemeinschaft under Grant No. Fr 737/2-2.

\section{APPENDIX: OFF-DIAGONAL MATRIX ELEMENTS OF THE DJS}

To compute the action of $\Sigma_{n<m}\left(S_{n}^{+} S_{m}^{-}+S_{n}^{-} S_{m}^{+}\right)$on the DJS (2.4) we define

$$
\left|\chi\left(\left\{n_{i}\right\}\right)\right\rangle=\prod_{i} S_{n_{i}}^{-}|\uparrow \cdots \uparrow\rangle
$$

with $\quad\left|\left\{n_{i}\right\}\right|=M . \quad$ Introducing $\quad z=e^{l(2 \pi / N)} \quad$ and $\quad \mu_{n_{i}}$ $=\left(z^{2 w}\right)^{(-1)^{n_{i}}}$ we find

$$
\begin{aligned}
& \frac{\left\langle\chi\left(\left\{n_{i}\right\}\right)\right| \Sigma_{n<m}\left(S_{n}^{+} S_{m}^{-}+S_{n}^{-} S_{m}^{+}\right) \psi^{N, M, J}(\theta, \rho)}{\left\langle\chi\left(\left\{n_{i}\right\}\right) \mid \psi^{N, M, J}(\theta, \rho)\right\rangle} \\
& \quad=\sum_{n=1}^{N / 2-1} z^{J 2 n} \sum_{i}\left(\prod_{\substack{i \neq \neq j \\
\left|n_{i}-n_{j}\right| \in 2 N}} \frac{\left(z^{n_{i}+2 n}-z^{n_{j}}\right)\left(z^{-n_{i}-2 n}-z^{-n_{j}}\right)}{\left(z^{n_{i}}-z^{n_{j}}\right)\left(z^{-n_{i}}-z^{-n_{j}}\right)} \prod_{\substack{i \neq j \\
\left|n_{i}-n_{j}\right| \notin 2 N}} \frac{\mu_{n_{i}} z^{n_{i}+2 n^{\prime}}-z^{n_{j}}}{\mu_{n_{i}} z^{n_{i}-} z^{n_{j}}} \frac{\left(1 / \mu_{n_{i}}\right) z^{-n_{i}-2 n}-z^{-n_{j}}}{\left(1 / \mu_{n_{i}}\right) z^{-n_{i}-} z^{-n_{j}}}\right)
\end{aligned}
$$




$$
\begin{aligned}
& +\sum_{n=0}^{N / 2-1} z^{J(2 n+1)} \sum_{i}\left(\prod_{\substack{i \neq j \\
\left|n_{i}-n_{j}\right| \in 2 N}} \frac{\left(1 / \mu_{n_{i}}\right) z^{n_{i}+2 n+1}-z^{n_{j}}}{z^{n_{i}-z^{n_{j}}}} \frac{\mu_{n_{i}} z^{-n_{i}-2 n-1}-z^{-n_{j}}}{z^{-n_{i}-z^{-n_{j}}}}\right. \\
& \left.\times \prod_{\substack{i \neq j \\
\left|n_{i}-n_{j}\right| \notin 2 N}} \frac{z^{n_{i}+2 n+1}-z^{n_{j}}}{\mu_{n_{i}} z^{n_{i}}-z^{n_{j}}} \frac{z^{-n_{i}-2 n-1}-z^{-n_{j}}}{\left(1 / \mu_{n_{i}}\right) z^{-n_{i}}-z^{-n_{j}}}\right) .
\end{aligned}
$$

The two sums originate from hopping terms between spins separated by an even and odd number of lattice sites, respectively. Combining these terms (A2) can be rewritten as

$$
\frac{\left\langle\chi\left(\left\{n_{i}\right\}\right)\right| \sum_{n<m}\left(S_{n}^{+} S_{m}^{-}+S_{n}^{-} S_{m}^{+}\right) \psi^{N, M, J}(\theta, \rho)}{\left\langle\chi\left(\left\{n_{i}\right\}\right) \mid \psi^{N, M, J}(\theta, \rho)\right\rangle}+M=\sum_{n=0}^{N-1} z^{n J} P\left[( \pm z)^{n},( \pm z)^{-n}\right],
$$

where $P$ is a polynomial in its arguments with coefficients independent of $n$. Only powers $( \pm z)^{n k}$ with $-(M-1) \leqslant k \leqslant(M-1)$ arise in the expansion (A3), so that for integer $J$ with $M \leqslant J \leqslant N-M$ the sum taken over each term of $P$ vanishes leading to

$$
\sum_{n<m}\left(S_{n}^{+} S_{m}^{-}+S_{n}^{-} S_{m}^{+}\right)\left|\psi^{N, M, J}(\theta, \rho)\right\rangle=-M\left|\psi^{N, M, J}(\theta, \rho)\right\rangle
$$

*Electronic address: frahm@itp.uni-hannover.de

†Present address: Physikalisches Institut, Universität Bayreuth, D-95440 Bayreuth, Germany; electronic address: John.Schliemann@theo.phy.uni-bayreuth.de

${ }^{1}$ M. Hase, I. Terasaki, and K. Uchinokura, Phys. Rev. Lett. 70, 3651 (1993); M. Hase, I. Terasaki, K. Uehinokura, M. Tokunaga, N. Miura, and H. Obara, Phys. Rev. B 48, 9116 (1993).

${ }^{2}$ J. E. Lorenzo, K. Hirota, G. Shirane, J. M. Tranquada, M. Hase, K. Uchinokura, H. Kojima, I. Tanaka, and Y. Shibuya, Phys. Rev. B 50, 1278 (1994).

${ }^{3}$ G. Castilla, S. Chakravarty, and V. J. Emery, Phys. Rev. Lett. 75, 1823 (1995).

${ }^{4}$ H. Bethe, Z. Phys. 71, 205 (1931).

${ }^{5}$ C. K. Majumdar and D. K. Ghosh, J. Math. Phys. (N.Y.) 10, 1388 (1969).

${ }^{6}$ I. Affleck, T. Kennedy, E. H. Lieb, and H. Tasaki, Commun. Math. Phys. 115, 477 (1988).

${ }^{7}$ I. Affleck, D. Gepner, H. J. Schulz, and T. Ziman, J. Phys. A 22, 511 (1989)

${ }^{8}$ K. Okamoto and K. Nomura, Phys. Lett. A 169, 433 (1992).

${ }^{9}$ S. R. White and I. Affleck, Phys. Rev. B 54, 9862 (1996).

${ }^{10}$ E. H. Lieb and B. Nachtergaele, Phys. Rev. B 51, 4777 (1995); M. Aizenman and B. Nachtergaele, Commun. Math. Phys. 164, 17 (1994).

${ }^{11}$ B. S. Shastry and B. Sutherland, Phys. Rev. Lett. 47, 964 (1981).

${ }^{12}$ T. Barnes, E. Dagotto, J. Riera, and E. S. Swanson, Phys. Rev. B 47, 3196 (1993); S. Gopalan, T. M. Rice, and M. Sigrist, ibid. 49, 8901 (1994).

${ }^{13}$ M. Azzouz and C. Bourbonnais, Phys. Rev. B 53, 5090 (1996); V. N. Muthukumar, C. Gros, W. Wenzel, R. Valenti, P. Lemmens, B. Eisener, G. Güntherodt, M. Weiden, C. Geibel, and F.
Steglich, ibid. 54, R9635 (1996); V. N. Muthukumar, C. Gros, R. Valenti, M. Weiden, C. Geibel, F. Steglich, P. Lemmens, M. Fischer, and G. Güntherodt, ibid. 55, 5944 (1997).

${ }^{14}$ T. A. Kaplan, P. Horsch, and P. Fulde, Phys. Rev. Lett. 49, 889 (1982).

${ }^{15}$ F. D. M. Haldane, Phys. Rev. Lett. 60, 635 (1988); B. S. Shastry, ibid. 60, 639 (1988).

${ }^{16}$ F. D. M. Haldane, Phys. Rev. Lett. 66, 1529 (1991).

${ }^{17}$ C. Zeng and J. B. Parkinson, Phys. Rev. B 51, 11609 (1995); S. Brehmer, H.-J. Mikeska, and U. Neugebauer, J. Phys. Condens. Matter 8, 7161 (1996); S. Yamamoto, Phys. Rev. B 55, 3603 (1997); D. Khomskii, W. Geertsma, and M. Mostovoy, Czech. J. Phys. 46, 3239 (1996).

${ }^{18}$ X. G. Wen, F. Wilczek, and A. Zee, Phys. Rev. B 39, 11413 (1989).

${ }^{19}$ F. Gebhard and D. Vollhardt, Phys. Rev. Lett. 59, 1472 (1987); Phys. Rev. B 38, 6911 (1988).

${ }^{20}$ H. Nakano and M. Takahashi, J. Phys. Soc. Jpn. 64, 2762 (1995).

${ }^{21}$ M. C. Gutzwiller, Phys. Rev. Lett. 10, 159 (1963).

${ }^{22}$ W. Metzner and D. Vollhardt, Phys. Rev. Lett. 59, 121 (1987); Phys. Rev. B 37, 7382 (1988).

${ }^{23}$ Y. Kuramoto and H. Yokoyama, Phys. Rev. Lett. 67, 1338 (1991); H. Yokoyama and M. Ogata, ibid. 67, 3610 (1991).

${ }^{24}$ J. Schliemann, Diploma thesis, Universität Hannover, 1996.

${ }^{25}$ R. Chitra, S. Pati, H. R. Krishnamurthy, D. Sen, and S. Ramasesha, Phys. Rev. B 52, 6581 (1995).

${ }^{26}$ T. Tonegawa and I. Harada, J. Phys. Soc. Jpn. 56, 2153 (1987).

${ }^{27}$ F. D. M. Haldane, Z. N. C. Ha, J. C. Talstra, D. Bernard, and V. Pasquier, Phys. Rev. Lett. 69, 2021 (1992).

${ }^{28}$ M. Hase, I. Terasaki, Y. Sasago, K. Uchinokura, and H. Obara, Phys. Rev. Lett. 71, 4059 (1993); Y. Sasago, N. Koide, K. Uchinokura, M. C. Martin, M. Hase, K. Hirota, and G. Shirane, Phys. Rev. B 54, R6835 (1996). 